\title{
Relação entre conforto térmico urbano e Zonas Climáticas Locais
}

\author{
Relationship between outdoor thermal comfort and Local Climate Zones
}

Maria Eugênia Fernandes[a] [], Érico Masiero[b] [1]

[a] Universidade Federal de São Carlos (UFSCar), Programa de Pós-graduação em Engenharia Urbana, São Carlos, SP, Brasil

[b] Universidade Federal de São Carlos (UFSCar), Departamento de Engenharia Civil, Programa de Pós-graduação em Engenharia Urbana, São Carlos, SP, Brasil

Como citar: Fernandes, M. E, \& Masiero, E. (2020). Relação entre conforto térmico urbano e Zonas Climáticas Locais. urbe. Revista Brasileira de Gestão Urbana, 12, e20190247. https://doi.org/10.1590/2175-3369.012.e20190247

\section{Resumo}

O ambiente urbano pode impactar nas condições de conforto térmico de acordo com a sua configuração, afetando o uso do espaço e a qualidade de vida da população. Assim, é importante conhecer o nível de conforto dos espaços urbanos e as consequências para a população para propor soluções construtivas adequadas. Este artigo objetiva analisar a influência da forma urbana e da cobertura do solo no conforto térmico dos usuários de espaços públicos ao ar livre. Para isso, foram caracterizadas quatro frações da cidade de São Carlos-SP utilizando o sistema de classificação das Zonas Climáticas Locais (ZCL). Foram realizadas coletas de dados microclimáticos e variáveis pessoais em três períodos para calcular o índice PET. Além disso, foram investigadas a percepção e a satisfação térmica dos usuários. Os resultados mostraram que o índice PET variou de $25^{\circ} \mathrm{C}$ a $48^{\circ} \mathrm{C}$, representando desconforto por calor em todos os pontos, também reforçado pelo registro da percepção dos usuários. No entanto, estes mostraram satisfação nos espaços de lazer, especialmente nos arborizados, mesmo em condições de elevado índice PET, o que indicaria desconforto por calor. As ZCL compactas e com grande percentual de impermeabilidade apresentaram as piores condições, registrando insatisfação dos usuários em todos os períodos.

Palavras-chave: Conforto térmico. Geometria urbana. Índice PET. Zonas Climáticas Locais

\section{Abstract}

The urban environment can impact thermal comfort conditions according to its configuration, affecting the use of space and the quality of life of the population. Thus, it is important to know the comfort level of urban spaces and the consequences for the population, in order to propose appropriate constructive solutions. This paper aims to analyze the influence of urban form and land cover on the thermal comfort of users of outdoor public spaces. For this, four fractions of the city of São Carlos-SP were characterized by the Local Climate Zones classification system. Microclimatic data and personal variables were collected in three periods to calculate the PET index. In addition, the perception and thermal satisfaction of users were

MEF é arquiteta e urbanista, mestre em Engenharia Urbana e doutoranda, e-mail: fernandes.me88@gmail.com

EM é arquiteto e urbanista, doutor em Engenharia Urbana e professor, e-mail: erico@ufscar.br 
investigated. The results show that the PET index ranged from $25^{\circ} \mathrm{C}$ to $48{ }^{\circ} \mathrm{C}$, representing heat discomfort at all points, also reinforced by the registration of users' perception. However, users show satisfaction in leisure spaces, especially in wooded spaces, even in conditions of high PET index, which would indicate heat discomfort. Compact ZCLs with a high percentage of impermeability present the worst conditions, registering user dissatisfaction in all periods.

Keywords: Thermal comfort. Urban geometry. PET index. Local Climate Zones.

\section{Introdução}

O processo de urbanização tem influenciado o comportamento climático, afetando a qualidade de vida da população. Algumas alterações na paisagem natural, como a redução das áreas verdes, a impermeabilização das superfícies urbanas por meio de pavimentação intensiva e a construção de edifícios, podem causar o aumento do calor antropogênico liberado para a atmosfera (Santamouris, 2015).

Um dos principais fenômenos causados pelas mudanças microclimáticas nas cidades são as Ilhas de Calor Urbana (ICU), notadas pelo excessivo aumento das temperaturas do ar nas áreas centrais das cidades em relação às do ambiente rural (Oke, 1987). Tal ocorrência, além de gerar desconforto térmico, pode impactar na saúde da população, levando à morte por doenças cardiovasculares e respiratórias (Sobral, 2005; Nobre, 2011).

Assim, é reforçada a necessidade de haver espaços urbanos adequados, capazes de reduzir as adversidades climáticas e oferecer ambientes que promovam qualidade de vida. Jamei et al. (2016) apontam a importância do planejamento urbano na promoção de conforto térmico na escala do pedestre por meio da integração entre as considerações climáticas e o desenho das cidades.

Kruger \& Drach (2017) mostram que o desconforto térmico em ambientes internos, que tem levado ao aumento do uso de ar-condicionado, apresenta influência também no comportamento do usuário em ambiente externo. A pesquisa indicou que pessoas habituadas ao uso de climatização artificial apresentam maior desconforto por calor no espaço aberto, concluindo que a aclimatação desses usuários é mais difícil tanto pelo histórico térmico quanto pela fisiologia. Os autores apontam ainda que esse fato resulta no aumento da pressão por soluções urbanísticas de mitigação de calor em espaços públicos.

Dessa forma, diversos estudos (Oke, 1981; Souza et al., 2010; Middel et al., 2014; Wei et al. 2016) têm buscado relacionar os parâmetros da forma urbana às variações climáticas e à formação das ICU, mostrando que a alteração desses elementos pode afetar o microclima.

Recentemente, Stewart \& Oke (2012) propuseram um sistema de classificação com base na análise da geometria urbana e na cobertura do solo. 0 sistema chamado de Zonas Climáticas Locais (ZCL) propõe a criação de um padrão para análise de estudos do clima urbano, permitindo a comparação entre eles. A metodologia tem se mostrado eficiente para explicar as variações térmicas em diferentes configurações urbanas (Perera \& Emmanuel, 2016; Alexander \& Mills, 2014), sendo uma importante ferramenta para planejar soluções que minimizem os impactos das adversidades climáticas e, consequentemente, o conforto térmico do pedestre.

Nesse contexto, diversos índices destinados à avaliação das condições de conforto térmico têm sido aplicados às cidades no mundo todo, buscando qualificar os espaços públicos abertos (Cheng et al., 2012; Givoni et al., 2003; Kruger \& Tamura, 2015; Ng \& Cheng, 2012).

Um dos índices mais utilizados pelas pesquisas para avaliar o conforto térmico em áreas urbanas abertas é o Physiological Equivalent Temperature (PET), ou Temperatura Fisiológica Equivalente, desenvolvido por Höppe (1999). Alguns estudos como os de Herrmann \& Matzarakis (2011) e Lee \& Mayer (2018) buscaram relacionar o PET à geometria, mostrando que, em grande parte dos casos, o desenho urbano pode influenciar no nível de conforto dos usuários.

Dada a importância de estudos que considerem a relação entre a geometria urbana e o conforto térmico para o desenvolvimento de diretrizes urbanas responsivas (Abreu-Harbich et al., 2013), este trabalho teve como objetivo analisar a influência da forma urbana e da cobertura do solo no conforto térmico dos usuários em quatro espaços públicos ao ar livre com diferentes configurações físicas na cidade de São Carlos-SP. 


\section{Método}

A presente pesquisa foi dividida em quatro etapas, descritas a seguir:

1. Definição e caracterização física dos espaços;

2. Coleta de dados microclimáticos e pessoais para cálculo do índice de conforto (PET);

3. Coleta de dados da percepção térmica e satisfação dos usuários;

4. Espacialização do nível de conforto térmico e estabelecimento de relações entre configurações morfológicas urbanas e condições de conforto térmico humano.

\section{Localização e escolha dos pontos}

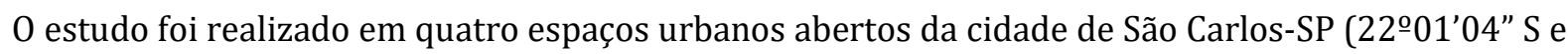
47-53'27" W), localizada a aproximadamente $228 \mathrm{~km}$ da capital, com uma altitude média de $856 \mathrm{~m}$ acima do nível do mar. 0 clima da cidade, de acordo com a classificação de Köpen-Geiger, é definido como Cwa (clima subtropical/clima tropical de altitude).

A escolha dos pontos atendeu aos seguintes critérios:

- Espaços públicos ao ar livre;

- Grande circulação de pessoas em diversos horários;

- Ocorrência recente de alterações do desenho urbano (últimos 10 anos).

Os quatro recortes analisados encontram-se delimitados na Figura 1.

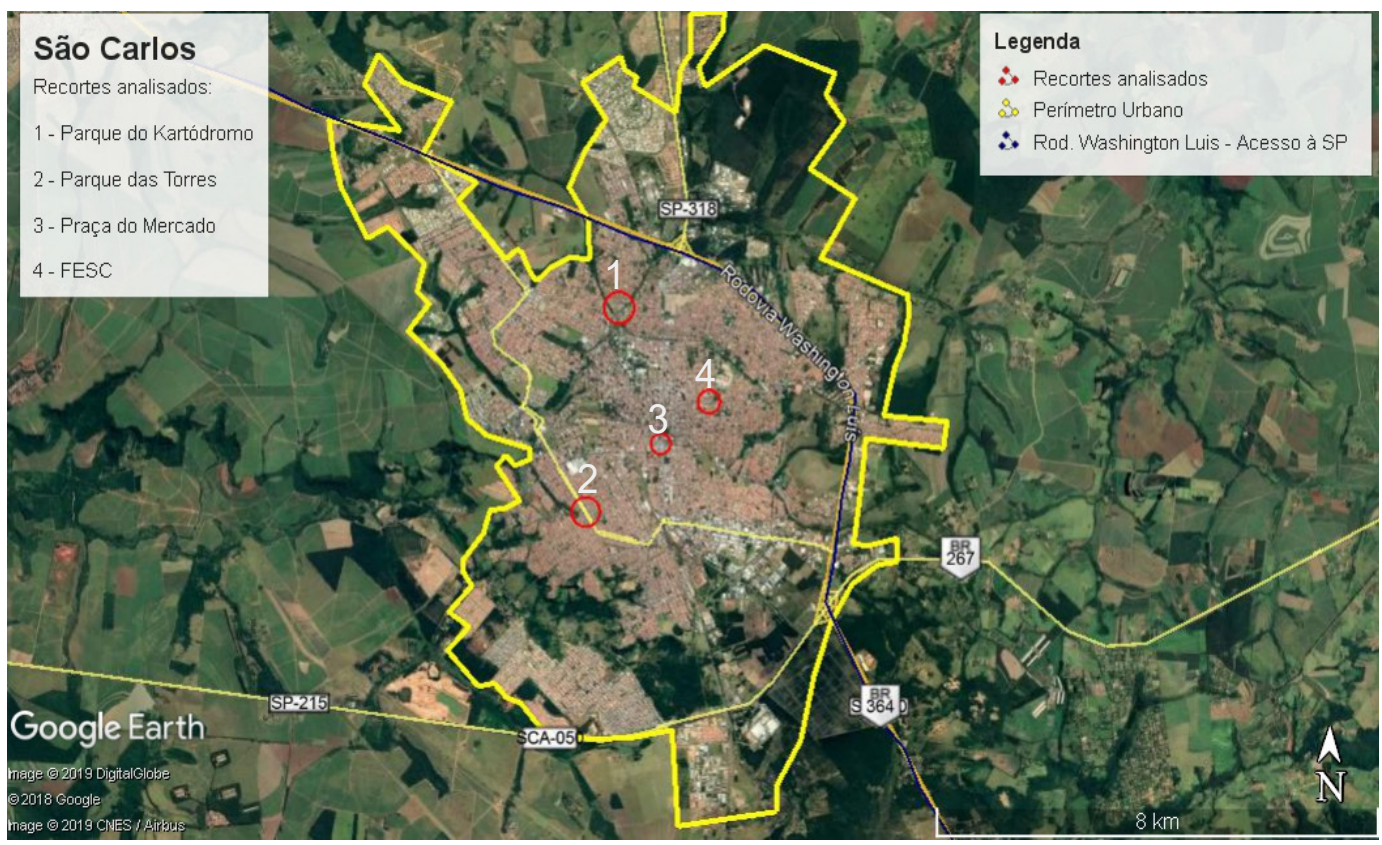

Figura 1 - Posição dos recortes urbanos analisados na cidade de São Carlos. Fonte: adaptada de Google Earth, versão 7.3.1.4507 (Google Inc., 2018), e de São Carlos (2016).

0 ponto 1, Parque do Kartódromo, localiza-se em um fundo de vale (altitude $811 \mathrm{~m}$ ), no encontro de dois córregos, com entorno bastante arborizado, em uma região essencialmente residencial. 0 parque foi construído sobre a antiga pista de kart da cidade, desativada em 2004. Atualmente, o local conta com 
pista de caminhada, parque infantil, academia ao ar livre e é utilizado para diversos eventos públicos, feiras e prática de atividades esportivas e de lazer ao ar livre em uma área de 22.620,00 m².

0 ponto 2, Parque das Torres (altitude $833 \mathrm{~m}$ ), é um parque linear situado no canteiro central de uma avenida de grande fluxo de veículos, possui uma ciclofaixa e pista de caminhada com extensão de 1,3 km, além de diversos equipamentos urbanos. 0 entorno do parque é formado por residências e estabelecimentos comerciais.

0 ponto 3, Praça do Mercado (altitude $809 \mathrm{~m}$ ), está localizado na região central da cidade, com intenso fluxo de veículos e pedestres, por causa da forte atividade comercial exercida nessa área. No entanto, o local já passou por diversas obras na tentativa de solucionar os constantes problemas de enchente enfrentados anualmente. Em 2012, o córrego ali existente foi tamponado, e, atualmente, o espaço abriga vários eventos culturais como shows e feiras.

O ponto 4, Fundação Educacional de São Carlos (FESC) (872 m), foi um campo de futebol que, após processo de revitalização, passou a ser utilizado para atividades educativas, esportivas e de lazer. Foram construídas salas de aula, piscina coberta, prédio administrativo, estacionamento, além de academia ao ar livre e parque infantil. 0 gramado do campo foi mantido, no entanto, sobre ele, foi construído um percurso para caminhada. Apesar de ser fechado, o local é de acesso público, com horário de funcionamento das $6 \mathrm{~h}$ às $22 \mathrm{~h}$ e possui grande demanda de visitantes.

A Figura 2 mostra cada um dos pontos analisados (em amarelo) e o recorte (em vermelho) que foi considerado no seu entorno para a análise das ZCL.
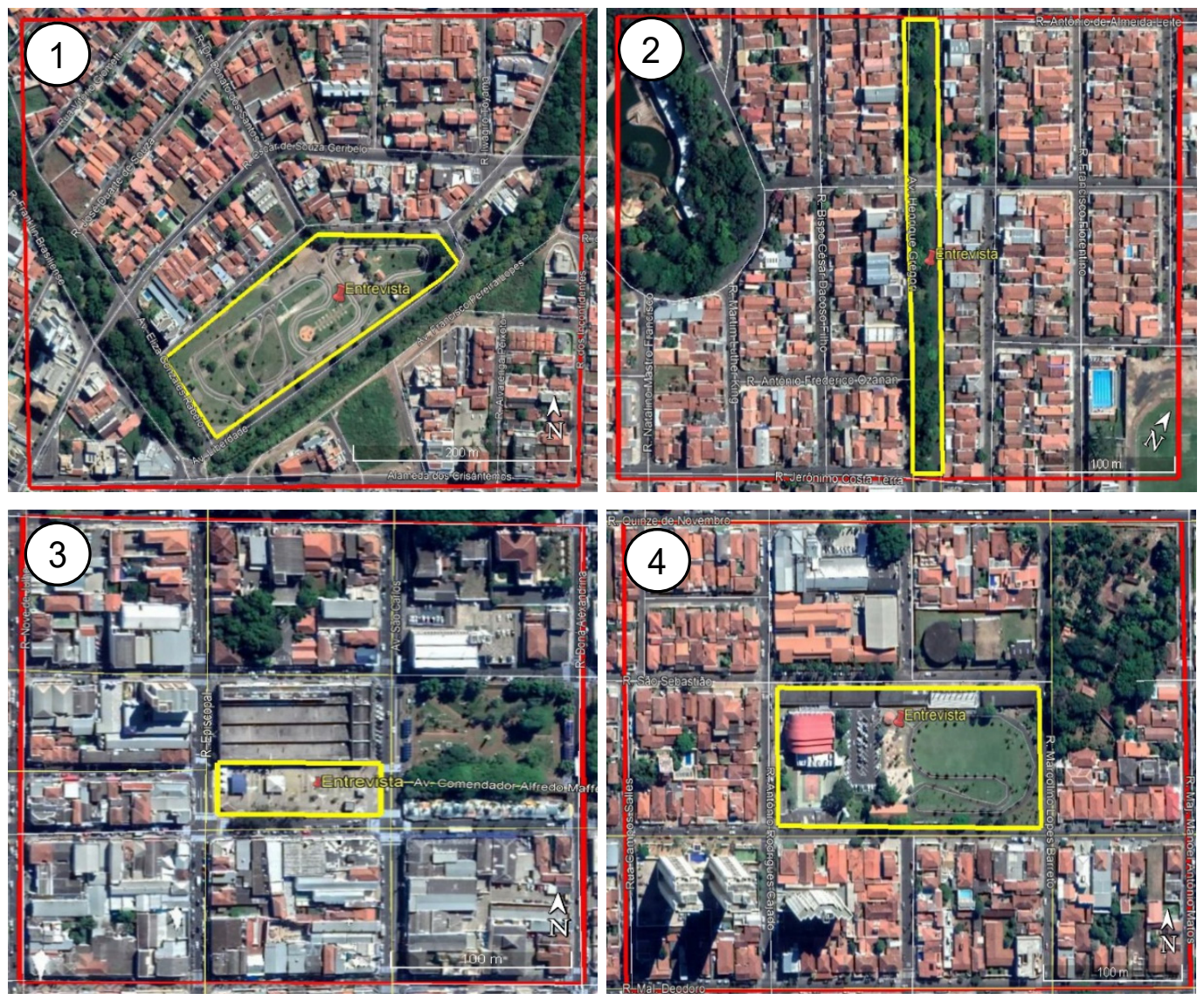

Figura 2 - Recortes urbanos analisados. 1 - Parque do Kartódromo; 2 - Parque das Torres; 3 - Praça do Mercado; 4 - Fundação Educacional de São Carlos (FESC). Fonte: adaptada de Google Earth, versão 7.3.1.4507 (Google Inc., 2018). 


\section{Caracterização física dos espaços}

A caracterização dos espaços se deu por meio da metodologia de classificação das Zonas Climáticas Locais (ZCL) apresentada por Stewart \& Oke (2012). Tal sistema se baseia na análise da tipologia das construções e da cobertura do solo por meio dos parâmetros descritos na Tabela 1.

Tabela 1 - Obtenção de dados para classificação das ZCL

\begin{tabular}{cc}
\hline Parâmetro & Forma de obtenção dos dados \\
\hline Porcentagem de área permeável & Imagem de satélite + AutoCAD ${ }^{\circledR}+$ confirmação em campo \\
Porcentagem de área impermeável & Imagem de satélite + AutoCAD ${ }^{\circledR}+$ confirmação em campo \\
Porcentagem de área construída & Imagem de satélite + AutoCAD ${ }^{\circledR}$ confirmação em campo \\
Fator de Visão do Céu (FVC) & Fotografia com lente olho de peixe + RayMan 1.2 \\
Relação H/W (altura/largura da via) & Google Street View + confirmação em campo \\
Altura média dos elementos & Google Street View + confirmação em campo \\
Classe de rugosidade & Davenport et al. (2000) \\
\hline
\end{tabular}

Fonte: elaborada pelos autores (2019).

As imagens de satélite foram obtidas do Google Earth (Google Inc., 2018) e transferidas para o programa Autocad $\AA$ com o intuito de obter informações da geometria urbana de cada elemento, tais como edifícios, gramados, árvores, calçadas e vias.

Após a obtenção dos dados, cada parâmetro da geometria urbana foi cruzado com os valores de referência estabelecidos por Stewart \& Oke (2012), e a classificação foi realizada segundo a correspondência dos valores obtidos com cada uma das classes.

\section{Análise do conforto térmico dos usuários}

A análise do conforto térmico foi dividida em duas etapas realizadas simultaneamente:

\section{Etapa a) Determinação do índice de conforto}

0 índice de conforto adotado para esta pesquisa foi Temperatura Fisiológica Equivalente (PET). De acordo com Höppe (1999), o índice PET é a temperatura do ar em que, em um ambiente interno, sem vento e radiação solar, o ganho de calor do corpo humano está equilibrado com a mesma temperatura da pele e do núcleo em condições iguais às de avaliação.

Para obtenção do PET, foi realizada a coleta de dados microclimáticos e dados pessoais dos indivíduos. A Tabela 2 ilustra as variáveis necessárias para esse cálculo e os instrumentos utilizados para sua obtenção.

Tabela 2 - Parâmetros necessários para o cálculo do índice PET

\begin{tabular}{cc}
\hline Variável & Forma de obtenção \\
\hline Temperatura do ar $\left({ }^{\circ} \mathrm{C}\right)$ & Termo-higrômetro HOBO Pro V2 U23-001 (1) \\
Umidade relativa do ar $(\%)$ & Termo-higrômetro HOBO Pro V2 U23-001 (1) \\
Temperatura do globo $\left({ }^{\circ} \mathrm{C}\right)$ & Termômetro de globo cinza (confeccionado com termoanemômetro Kimo \\
Velocidade do vento $(\mathrm{m} / \mathrm{s})$ & VT200 + bola de pingue-pongue (D = 40 mm) pintada na cor cinza médio) (2) \\
Sexo, idade, peso $(\mathrm{kg})$ e altura $(\mathrm{m})$ & Termoanemômetro Digital Instrutherm TAVR - 650 (3) \\
Coeficiente de isolamento térmico & Entrevista estruturada \\
da vestimenta (CLO) & Entrevista + valores estabelecidos na ISO 7730/2005 (ISO, 2005) \\
Taxa metabólica (MET) & Entrevista + valores estabelecidos na ISO 7730/2005 (ISO, 2005) \\
\hline
\end{tabular}

Fonte: elaborada pelos autores (2019).

Os equipamentos foram posicionados em um suporte metálico, a aproximadamente 1,5 $\mathrm{m}$ do solo (Figura 3), e as medições foram registradas a cada minuto. 


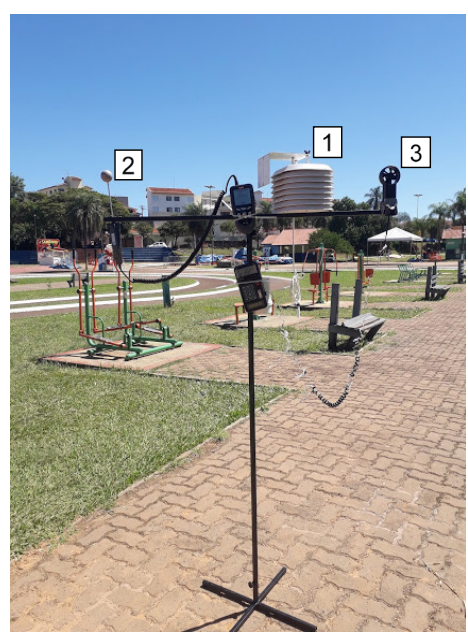

Figura 3 - Equipamentos utilizados para medição. 1 - Termo-higrômetro HOBO Pro V2 U23-001; 2 - Termômetro de globo cinza; 3 - Termoanemômetro Digital Instrutherm TAVR - 650. Fonte: fotografia tirada pelos autores (2019).

Para calcular o valor da temperatura média radiante (TRM), foi adotada a equação de convecção forçada (Equação 1), conforme indicado pela ISO 7726 (ISO, 1998):

$\operatorname{Trm}=\left[(\operatorname{tg}+273)^{4}+\frac{1,1 \times 10^{8} \times \mathrm{Va}^{0,6}}{\operatorname{Eg} \times D^{0,4}} \times|\operatorname{tg}-\operatorname{ta}|^{\frac{1}{4}}-273\right.$

Em que:

$\operatorname{tg}=$ temperatura do globo $\left[{ }^{\circ} \mathrm{C}\right]$.

$\mathrm{Va}=$ velocidade do ar $[\mathrm{m} / \mathrm{s}]$.

$\mathrm{Eg}=$ emissividade do globo.

$\mathrm{D}=$ diâmetro do globo $[\mathrm{m}]$.

ta $=$ temperatura do ar $\left[{ }^{\circ} \mathrm{C}\right]$.

O cálculo do índice PET foi realizado utilizando o software RayMan 1.2 (Rutz et al., 2000) e considerou as variáveis pessoais declaradas pelos usuários, bem como os dados microclimáticos coletados.

\section{Etapa b) Análise da percepção térmica e da satisfação térmica dos usuários}

As entrevistas também analisaram a percepção térmica dos usuários por meio da escala de 7 pontos e a satisfação térmica mediante a escala de 5 pontos, conforme recomendação da ISO 10551 (ISO, 1995) e da ASHRAE 55 (ASHRAE, 2004) (Figura 4).

$\begin{aligned} & \text { Como você está se sentindo nesse } \\ & \text { momento com relação à temperatura do } \\ & \text { ar? }\end{aligned}$
frio

\begin{tabular}{|c|c|c|c|c|c|c|}
\hline $\begin{array}{l}\text { Quão satisfeito você está com a } \\
\text { temperatura nesse ambiente? }\end{array}$ & $\begin{array}{l}\text { Muito } \\
\text { satisfeito }\end{array}$ & Satisfeito & Indiferente & Insatisfeito & Morno & $\begin{array}{c}\text { Muito } \\
\text { insatisfeito }\end{array}$ \\
\hline
\end{tabular}

Figura 4 - Questionário adotado para investigação da percepção e satisfação térmica do usuário. Fonte: elaborada pelos autores (2019).

As etapas a e b foram realizadas entre dezembro de 2018 e janeiro de 2019, durante o verão, em dias de tempo estável, ventos fracos, com poucas ou nenhumas nuvens. Foram realizados dois dias de coleta em cada um dos pontos, executados em três diferentes períodos, sendo eles: das $10 \mathrm{~h}$ às $11 \mathrm{~h}$, das $16 \mathrm{~h}$ às $17 \mathrm{~h}$ e das $19 \mathrm{~h}$ às $20 \mathrm{~h}$, considerando o horário brasileiro de verão (GMT -2). 
A amostragem adotada em ambas as etapas foi definida em função do maior número de respondentes em cada período. Dessa forma, foram realizadas 325 entrevistas, das quais $60 \%$ pertenciam ao sexo masculino. A idade média encontrada entre os respondentes foi de 39 anos, com peso de $70 \mathrm{~kg}$ e altura $1,70 \mathrm{~m}$.

\section{Espacialização do nível de conforto térmico}

Com o objetivo de visualizar a distribuição dos níveis de conforto na malha urbana, foram gerados três mapas de temperatura PET para as $10 \mathrm{~h}, 16 \mathrm{~h}$ e $19 \mathrm{~h}$. Os dados utilizados para a espacialização foram obtidos por meio das médias dos valores de PET em cada ponto.

Os valores das médias foram inseridos no software Quantum GIS, versão 3.2.1, um Sistema de Informação Geográfica (SIG). 0 método escolhido para a criação dos mapas foi a Interpolação IDW (Inverse Distance Weighting, ou Ponderação do Inverso da Distância). No entanto, foi feita a opção por um recorte que abrangesse somente as áreas adjacentes aos pontos, buscando manter maior precisão estatística, já que a análise aqui realizada contemplou somente quatro pontos, e o IDW fundamenta-se na distância entre eles.

\section{Resultados}

\section{Zona climática local}

A Tabela 3 mostra os valores identificados para cada um dos parâmetros nas frações urbanas analisadas, bem como suas respectivas zonas climáticas.

Tabela 3 - Classificação das Zonas Climáticas Locais - ZCL

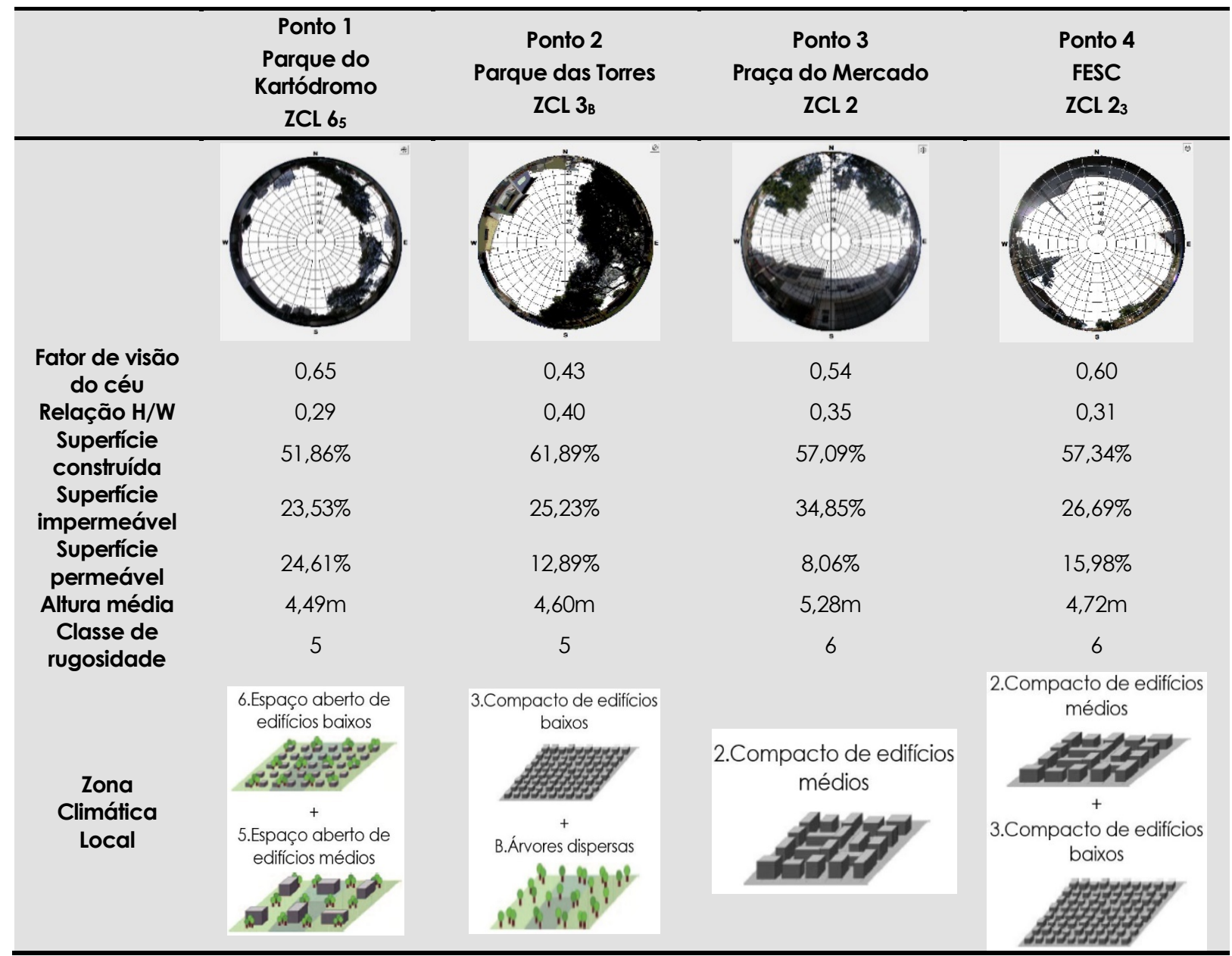

Fonte: elaborada pelos autores (2019). 
Por causa da falta de homogeneidade das áreas, foi necessário realizar a subclassificação para que as ZCL correspondessem melhor à situação real.

0 Parque do Kartódromo foi classificado como ZCL $6_{5}$ (espaço aberto de edifícios baixos e médios), cujas características sugerem regiões abertas com abundância de vegetação, edificações de baixo (1-3 pavimentos) e médio gabarito (1-9 pavimentos) e materiais de construção de concreto, aço, pedra e vidro.

O Parque das Torres (ZCL $3_{\mathrm{B}}$ - compacto de edifícios baixos com árvores dispersas) possui grande porcentagem de superfície construída de baixo gabarito (1-3 pavimentos), com cobertura do solo essencialmente pavimentada e presença de materiais de construção de concreto, aço, pedra e vidro, além de paisagem levemente arborizada.

A Praça do Mercado (ZCL 2 - compacto de edifícios médios) apresenta grande porcentagem de área impermeável e construída, edificações médias (1-9 pavimentos), cobertura do solo essencialmente pavimentada, com presença de materiais de construção de concreto, aço, pedra e vidro, assim como poucas vegetações.

A FESC está na ZCL $2_{3}$ (compacto de edifícios médios e baixos), com grande quantidade de área construída e impermeável, com edificações baixas e médias, presença de materiais de construção de concreto, aço, pedra e vidro, além de poucas vegetações.

Todas as frações analisadas possuíam mais de $50 \%$ de superfície construída, com destaque para a ZCL $3_{\mathrm{B}}$, com $10 \%$ a mais de área construída que a ZCL 65 . Por ser uma região ainda não totalmente consolidada, o entorno do Parque do Kartódromo possui muitos terrenos vazios e área de vegetação nas margens do córrego, o que também faz com que essa seja uma região mais aberta $(\mathrm{FVC}=0,65)$ e de maior porcentagem de área permeável $(24,61 \%)$.

Por outro lado, as ZCL 2 e 23 pertencem à regiões da cidade já mais consolidadas e compactas, ainda que apresentem FVC acima de 0,5, por causa da presença de algumas vias com grande largura, o que também faz com que as áreas apresentem semelhante relação $\mathrm{H} / \mathrm{W}(0,35$ e 0,31 , respectivamente).

As ZCL possuem pouca verticalização, sendo a maior altura média observada na Praça do Mercado (5,28 m) e a menor no Parque do Kartódromo (4,49 m).

Mesmo com a subclassificação, é possível perceber que nem todos os parâmetros se encaixam em uma mesma ZCL, sendo necessário adotar a que possui maior correspondência. Além disso, foi observado que, embora alguns pontos possuam grande diferença visualmente, os parâmetros têm valores semelhantes em ambas as regiões.

\section{Conforto térmico}

$\mathrm{O}$ índice PET variou de $25^{\circ} \mathrm{C}$ a $48^{\circ} \mathrm{C}$ nos três períodos analisados. A calibração proposta por Matzarakis et al. (2000) indica que os resultados estão acima do limite de conforto (18으 a $23^{\circ} \mathrm{C}$ ). No entanto, Labaki et al. (2012) analisaram o conforto térmico em outras cidades do interior de São Paulo e concluíram que a calibração de Monteiro \& Alucci (2007) se adequa melhor a essas situações. Dessa forma, os resultados aqui encontrados foram analisados de acordo com as faixas adotadas para a cidade de São Paulo (Monteiro \& Alucci, 2007). 


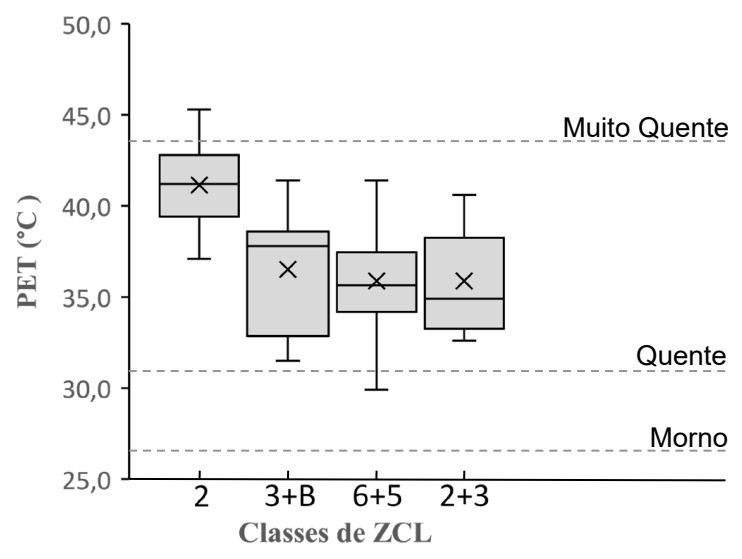

Figura 5 - Distribuição do conforto térmico para o período das 10h. Fonte: elaborada pelos autores (2019).

As ZCL $3_{\mathrm{B}}$ e $2{ }_{3}$ mostraram percepção de "Quente" no período da manhã. Nas ZCL 2 e 65 , mais de 75\% dos usuários encontravam-se na faixa "Quente", entre $31^{\circ} \mathrm{C}$ e $43^{\circ} \mathrm{C}$. No entanto, a ZCL 2 apresentou valores de PET mais altos que as demais, atingindo a faixa de percepção "Muito Quente", acima de $43^{\circ} \mathrm{C}$. A ZCL 65 mostrou uma grande variação, porém aproximadamente $75 \%$ dos usuários estavam mais próximos da faixa “Morno" do que da "Muito Quente" (Figura 5).

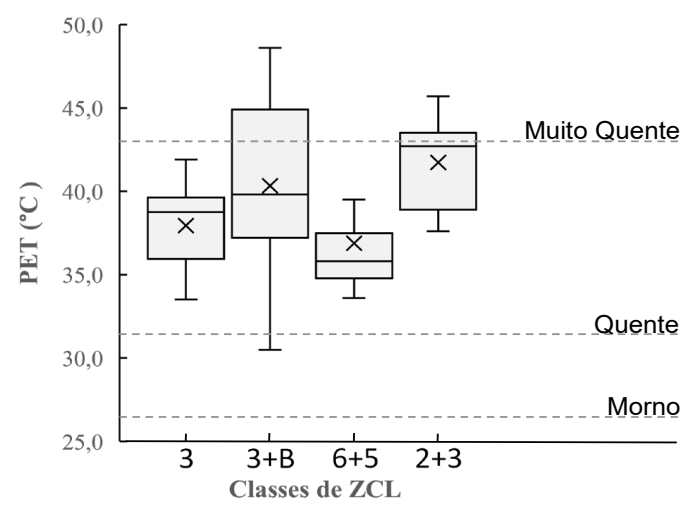

Figura 6 - Distribuição do conforto térmico para o período das 16h. Fonte: elaborada pelos autores (2019).

Às $16 \mathrm{~h}$, as ZCL $3_{\mathrm{B}}$ e $2_{3}$ apresentavam um aumento de desconforto, atingindo em mais de $25 \%$ a faixa de "Muito Quente", enquanto as ZCL 2 e $6_{5}$ se concentraram na faixa de percepção "Quente", mostrando uma queda no índice da ZCL 2 e uma homogeneidade maior na 65 (Figura 6).

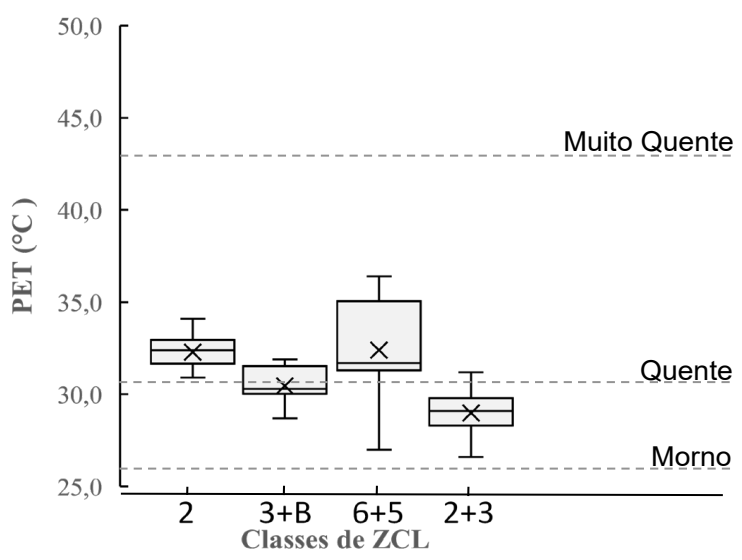

Figura 7 - Distribuição do conforto térmico para o período das 19h. Fonte: elaborada pelos autores (2019). 
Todas as ZCL apresentaram uma queda no período das $19 \mathrm{~h}$, sendo a maior registrada na $2_{3}$, com quase $100 \%$ dos usuários na faixa "Morno", enquanto, na 2, quase sua totalidade se encontrou na faixa "Quente". Mais de 50\% da ZCL $3_{\text {B }}$ estavam na faixa "Morno", e, na ZCL 65, aproximadamente $75 \%$ estavam na faixa "Quente" (Figura 7).

No horário da manhã, a ZCL 2 apresentou o pior desempenho, o que era esperado por causa da grande quantidade de área impermeável e construída, além da ausência de vegetação e elementos de sombreamento. Às $16 \mathrm{~h}$, no entanto, houve uma pequena redução no valor de PET nessa zona climática. Esse resultado pode ser favorecido pelo sombreamento, já que é o ponto com maior altura média dos elementos, especialmente em função da altura das edificações em quase toda a ZCL.

A ZCL 23, ainda que apresente parâmetros semelhantes aos da ZCL 2, mostrou comportamento inverso. Tal fato talvez se deva à diferença de altitude entre as regiões, já que estão, respectivamente, no ponto mais alto e mais baixo, com $63 \mathrm{~m}$ de diferença entre eles. Além disso, esse espaço, mesmo com ausência de arborização, conta com o sombreamento gerado pelas edificações, dependendo da posição do sol, o que poderia explicar a oscilação nos valores de PET.

0 mesmo ocorre com a ZCL $3_{B}$, que, inclusive, apresentou resultados muito parecidos com a ZCL $2_{3}$. 0 Parque das Torres é um parque linear arborizado, no entanto possui diversas clareiras, recebendo luz solar direta, além de estar entre duas vias de grande fluxo de veículos, o que aumenta o calor produzido na região. Por outro lado, a presença de edificações faz com que haja sombreamento em alguns períodos.

A ZCL $6_{5}$ apresentou resultados semelhantes nos períodos das $10 \mathrm{~h}$ e $16 \mathrm{~h}$, com os valores mais baixos alcançados. Porém, foi observada uma elevação nos valores de PET para as 19h. Embora esse ponto tenha grande quantidade de vegetação, as medições foram realizadas em um espaço aberto, com arborização no entorno, mas que não fornece sombreamento suficiente. Dessa forma, a incidência direta de sol durante todo o dia pode ter resultado nesse aumento de desconforto no início da noite.

Os resultados corroboram as pesquisas de Unger et al. (2018), na Hungria, e de Lau et al. (2019), em Hong Kong, que mostraram que as ZCL com maior percentual de área vegetada e arborização oferecem melhores condições de conforto, especialmente durante o dia.

As áreas arborizadas contêm um grande potencial para reduzir a temperatura do ar e aumentar a umidade por meio da evapotranspiração (Ali \& Patnaik, 2018). Além disso, essas áreas podem também gerar redução da TRM (Lindberg et al., 2016; Aminipouri et al., 2019), diminuindo o valor de PET.

Além de reduzir a temperatura do ar, o sombreamento pode gerar grande redução na temperatura dos pavimentos (Tsoka, 2017) e auxiliar na mitigação dos efeitos do aquecimento, produzindo espaços termicamente confortáveis (Middel et al., 2016).

\section{Percepção e satisfação térmica}

As Figuras 8, 9 e 10 mostram o resultado da análise dos votos de percepção e satisfação térmica dos usuários.
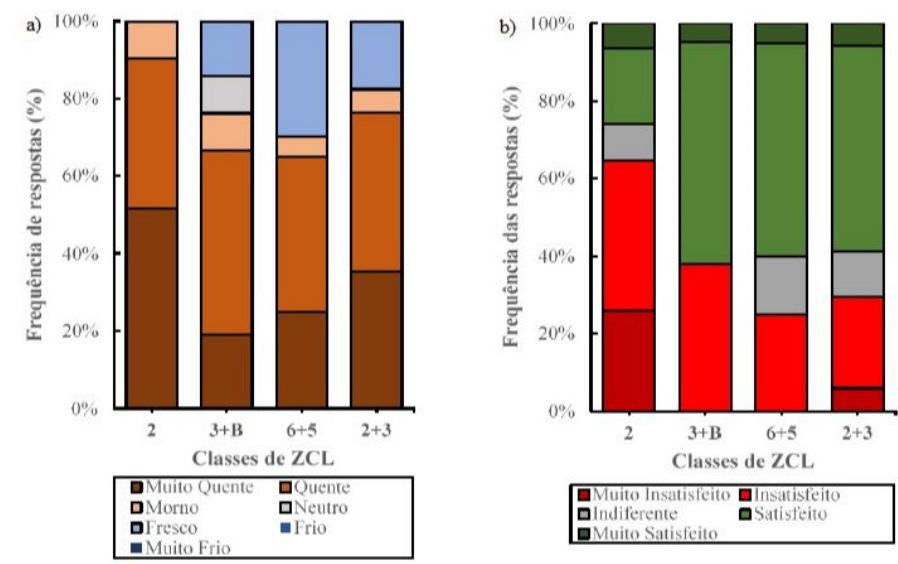

Figura 8 - (a) Percepção e (b) Satisfação térmica dos usuários para o período das 10h. Fonte: elaborada pelos autores (2019). 
Todas as ZCL apresentaram grande percepção de calor, no entanto, na ZCL 2, mais de 50\% dos usuários indicaram percepção de "Muito Quente"; nas demais prevaleceu "Quente" no período das 10h (Figura 8a).

Da mesma forma, a ZCL 2 apresentou a maior porcentagem de usuários insatisfeitos (65\%). Porém, nos outros pontos, aproximadamente $60 \%$ dos usuários se mostraram satisfeitos mesmo em condições de desconforto por calor (Figura 8b).
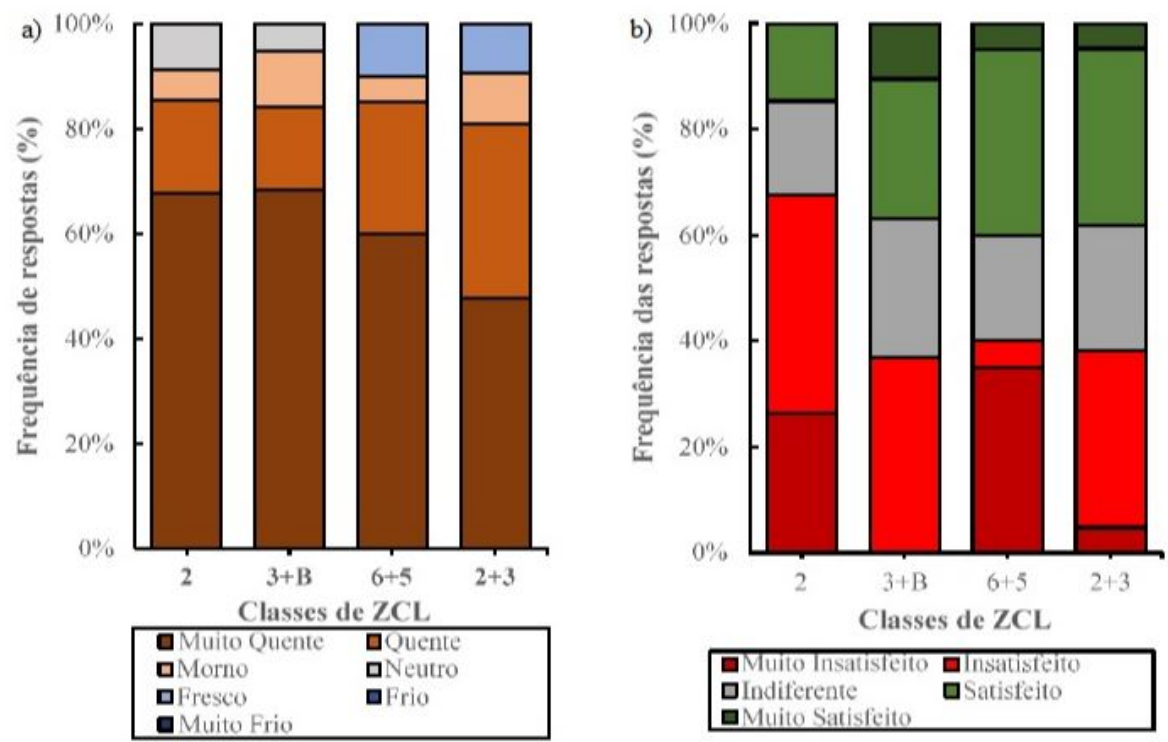

Figura 9 - (a) Percepção e (b) Satisfação térmica dos usuários para o período das 16h. Fonte: elaborada pelos autores (2019).

Às 16h, a percepção de "Muito Quente" cresceu em todas as ZCL, e os votos de desconforto ultrapassaram $80 \%$. Nas ZCL 2 e $3_{\mathrm{B}}$, respectivamente, $9 \%$ e $5 \%$ se declararam em neutralidade térmica (Figura 9a).

Por causa do aumento do desconforto, a satisfação térmica dos usuários diminuiu nesse horário. (Figura 9b). A insatisfação se manteve acima dos $60 \%$ na ZCL 2, enquanto nos outros pontos chegou a aproximadamente $40 \%$.
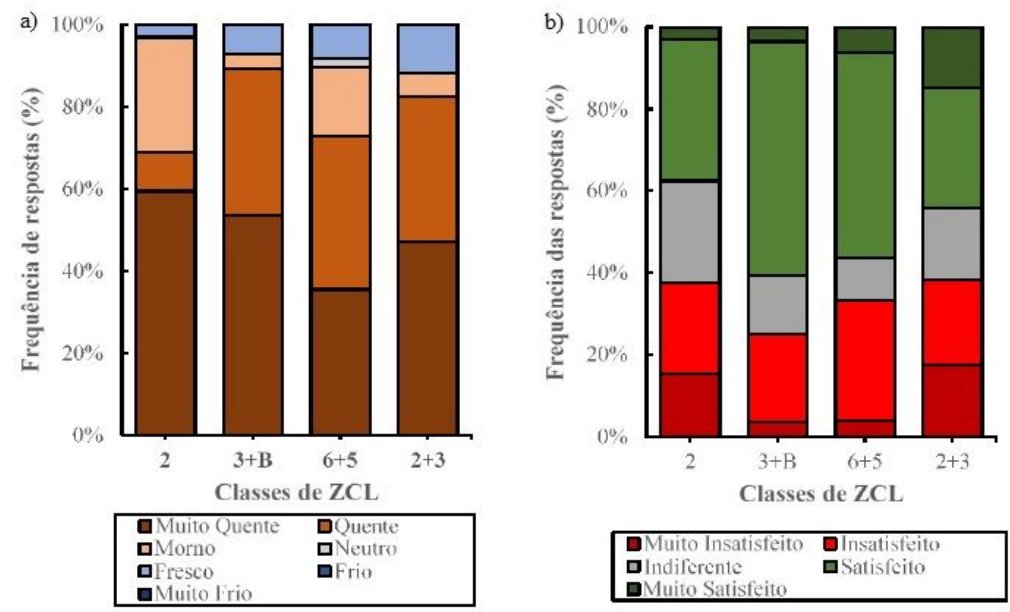

Figura 10 - (a) Percepção e (b) Satisfação térmica dos usuários para o período das 19h. Fonte: elaborada pelos autores (2019). 
O horário das 19h mostrou uma queda na quantidade de respostas para "Quente" e "Muito Quente", porém o desconforto por calor prevaleceu, ultrapassando $50 \%$ dos votos em todas as ZCL (Figura 10a). Na ZCL $3_{B}$, ainda que a percepção de "Muito Quente" tenha diminuído, o número de usuários fora da faixa de conforto continuou ultrapassando os $80 \%$.

Mesmo com grande percepção de desconforto térmico, a ZCL $3_{\mathrm{B}}$ foi o recorte em que os usuários mais se mostraram satisfeitos (61\%) nesse horário (Figura 10b). Na ZCL 2, ainda que a satisfação tenha aumentado (37\%), houve um equilíbrio com os votos de insatisfação (38\%). Na ZCL $6_{5}$, a satisfação foi indicada por $56 \%$ dos usuários, e na 23 , por $44 \%$.

Analogamente ao apresentado pelo índice PET, a percepção relatada pelos usuários indicou grande desconforto por calor, especialmente às 16h. Nesse horário, apesar da percepção de calor, foi observado um equilíbrio entre os votos de satisfação e insatisfação nos pontos analisados, com exceção da ZCL 2, em que a insatisfação prevaleceu nos três períodos.

Unger et al. (2018) e Lau et al. (2019) também mostraram que as ZCL com densa mistura de edifícios, grande taxa de impermeabilização e poucas árvores são capazes de gerar grande percepção de desconforto nos usuários.

Por outro lado, a ZCL 65, com maior percentagem de área permeável, espaços mais abertos e arborizados apresentou menor percepção de calor, além de grande satisfação, mesmo no período de maior desconforto.

Além da arborização, os equipamentos urbanos (bancos, lixeiras, bebedouro etc.) e o uso do espaço podem interferir na satisfação dos usuários (Labaki et al., 2012; Brusantin \& Fontes, 2009; Faustini \& Fontes, 2010). Basantes \& García (2018) apontaram que o tempo de permanência dos usuários é também um indicador de qualidade do lugar e que não depende somente das variáveis climáticas, mas de outros fatores como a atividade que ali realizam. Assim, as ZCL $3_{\mathrm{B}}, 6_{5}$ e $2_{3}$, por se tratarem de espaços de lazer, indicaram maior satisfação dos usuários, enquanto a ZCL 2, por estar situada em região de comércio, fez com que os pedestres estivessem ali por necessidade (trabalho ou compras), apresentando maior insatisfação com o ambiente.

\section{Espacialização do nível de conforto térmico}

A Figura 11 mostra a distribuição dos valores médios de PET na malha urbana para os três períodos. Foi observado que, nos períodos de temperaturas mais altas (10h e 16h), as maiores médias foram encontradas nos pontos próximos à área central da cidade, com entorno bastante construído. Ainda que se perceba uma diminuição no valor médio de PET para as 19h, é importante notar que todos os pontos permanecem fora da faixa de conforto.

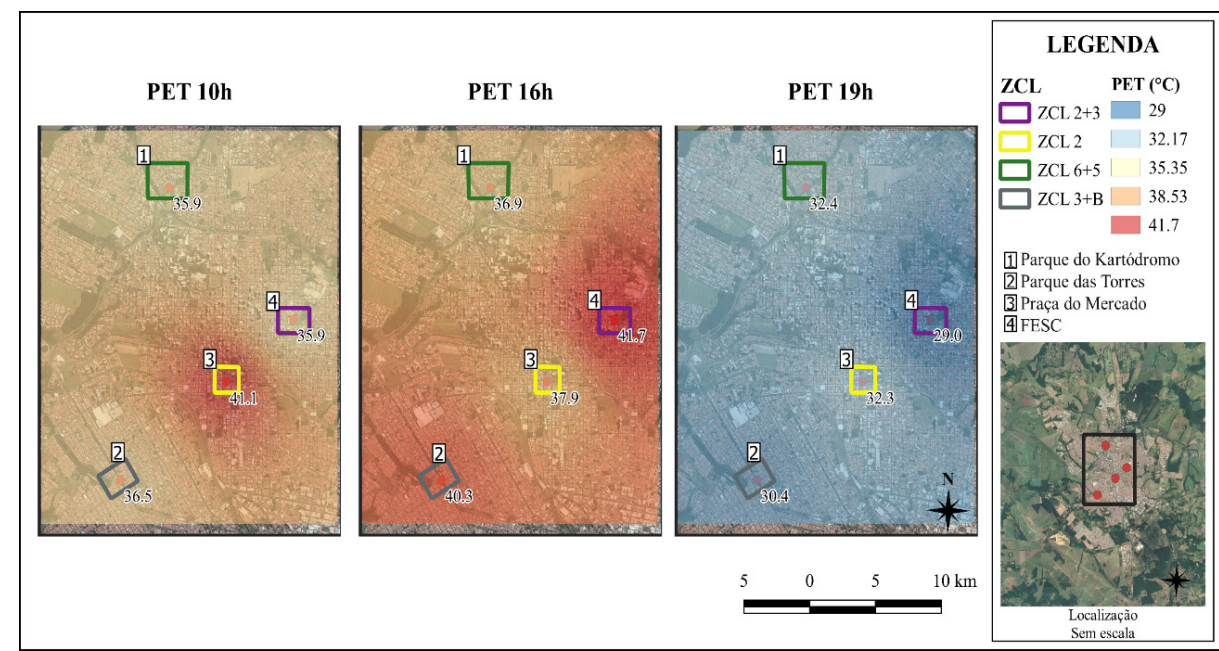

Figura 11 - Espacialização dos valores médios de PET para as 10h, 16h e 19h. Fonte: elaborada pelos autores (2019). 
Os quatro recortes estão inseridos em zonas urbanas em que o Plano Diretor Estratégico da cidade estabelece como coeficientes de ocupação e de permeabilidade 70\% e 15\%, respectivamente (São Carlos, 2016). Algumas pesquisas (Middel et al., 2014; Muniz-Gäal et al., 2018) mostram que o adensamento pode promover boas condições de conforto, na medida em que produz sombreamento e auxilia no controle do fluxo de vento. No entanto, o excesso de impermeabilidade do solo e a área construída tendem a elevar as temperaturas. Skarbit et al. (2017) revelaram que uma ZCL compacta, com grande quantidade de área impermeável, pode apresentar temperaturas cerca de $4^{\circ} \mathrm{C}$ mais quentes que as ZCL abertas e com vegetação.

Além disso, as áreas centrais tendem a ser mais quentes que as áreas periféricas, visto que estas têm maior proximidade com as áreas vegetadas e recebem maior quantidade de vento, por possuírem menos obstruções (Skarbit et al., 2017). Ferreira et al. (2017) também observaram que a região central de Belo Horizonte, área densamente ocupada por edificações, tem baixa capacidade de resfriamento noturno, visto que a carga térmica acumulada e o baixo potencial dinâmico apresentados favorecem o aquecimento das superfícies. Os autores alertam para os riscos de comprometimento ainda maior do clima urbano, visto que a legislação permite maior adensamento construtivo e verticalização, podendo resultar no aumento de carga térmica e na dificuldade de resfriamento, levando ao desconforto térmico.

Dessa forma, é importante que o planejamento urbano considere estratégias que busquem a integração entre o adensamento e a criação de áreas verdes, com vegetação e arborização suficientes para favorecer melhores condições climáticas e de conforto térmico (Masiero \& Souza, 2018).

\section{Conclusão}

A análise da relação entre as ZCL e o conforto térmico, além de apresentar a influência dos parâmetros urbanos no conforto do usuário, também confirma que a classificação das áreas pelo sistema das ZCL permite compreender as variações no nível de conforto térmico, podendo ser adotada para proposição de espaços urbanos com maior qualidade.

Embora os quatro espaços demonstrem desconforto por calor, foi observado que as zonas climáticas mais abertas e com maior quantidade de vegetação proporcionavam melhores condições de conforto, diferentemente dos espaços com alta taxa de impermeabilidade e grandes áreas construídas.

Foi constatado também que o tipo de uso/atividade pode influenciar na satisfação do usuário com determinado espaço. Sendo assim, é possível que os usuários se sintam satisfeitos em ambientes de lazer, mesmo em condição de desconforto térmico.

Tais resultados permitem auxiliar o planejamento da cidade, de forma a promover espaços com melhor qualidade de vida e gerar reflexões sobre a eficiência da legislação urbanística municipal, a fim de propor índices mais condizentes com as necessidades climáticas.

É recomendado que estudos futuros analisem o conforto térmico de usuários em diferentes configurações de ZCL e em outras estações do ano. Seria importante também determinar, por exemplo, o índice PET em condições de tempo frio e realizar sua calibração para a cidade de São Carlos-SP.

\section{Agradecimentos}

Os autores agradecem à Coordenação de Aperfeiçoamento de Pessoal de Nível Superior (CAPES) pela bolsa de mestrado concedida. 


\section{Referências}

Abreu-Harbich, L. V., Labaki, L. C., \& Matzarakis, A. (2013). Thermal bioclimate in idealized urban street canyons in Campinas, Brazil. Theoretical and Applied Climatology, 115(1-2), 333-340. http://dx.doi.org/10.1007/s00704-0130886-0.

Alexander, P., \& Mills, G. (2014). Local Climate Classification and Dublin's Urban Heat Island. Atmosphere., 5(4), 755-744. http://dx.doi.org/10.3390/atmos5040755.

Ali, S. B., \& Patnaik, S. (2018). Thermal comfort in urban open spaces: objective assessment and subjective perception study in tropical city of Bhopal, India. Urban Climate, 24, 954-967. http://dx.doi.org/10.1016/j.uclim.2017.11.006.

American Society of Heating Refrigerating, Airconditioning Engineers - ASHRAE. (2004). Standard 55: thermal environmental conditions for human occupancy. Atlanta: ASHRAE.

Aminipouri, M., Knudby, A. J., Krayenhoff, E. S., Zickfeld, K., \& Middel, A. (2019). Modelling the impact of increased street tree cover on mean radiant temperature across Vancouver's local climate zones. Urban Forestry \& Urban Greening, 39, 9-17. http://dx.doi.org/10.1016/j.ufug.2019.01.016.

Basantes, A. C. N., \& García, E. H. (2018). Altitud, variables climáticas y tiempo de permanencia de las personas en plazas de Ecuador. Urbe. Revista Brasileira de Gestão Urbana, 10(2), 414-425. http://dx.doi.org/10.1590/21753369.010.002.ao11.

Brusantin, G. N., \& Fontes, M. S. G. C. (2009). Conforto térmico. Espaços públicos de permanência: uma experiência na cidade de Bauru-SP. In Anais do X Encontro Nacional e VI Encontro Latino-americano sobre Conforto no Ambiente Construído (pp. 441-449.). Natal: ANTAC.

Cheng, V., Ng, E., Chan, C., \& Givoni, B. (2012). Outdoor thermal comfort study in a sub-tropical climate: A longitudinal study based in Hong Kong. International Journal of Biometeorology, 56(1), 43-56. http://dx.doi.org/10.1007/s00484-010-0396-z. PMid:21197549.

Davenport, A. G., Grimmond, C. S. B., Oke, T. R., \& Wieringa, J. (2000). Estimating the roughness of cities and sheltered country. In 15th conference on probability and statistics in the atmospheric sciences/12th conference on applied climatology (pp. 96-99). Ashville, NC: American Meteorological Society.

Faustini, B. F., \& Fontes, M. S. G. C. (2010). Conforto térmico em espaços públicos de passagem: estudos no calçadão da Batista de Carvalho em Bauru-SP, Brasil. In Anais do IX Congresso Luso-Brasileiro para o Planejamento Urbano, Regional, Integrado, Sustentável - PLURIS 2010 (Faro, Portugal). São Carlos: EESC-USP.

Ferreira, D. G., Assis, E. S., \& Katzschner, L. (2017). Construção de um mapa climático analítico para a cidade de Belo Horizonte, Brasil. Urbe. Revista Brasileira de Gestão Urbana, 9(1, suppl 1), 255-270.

http://dx.doi.org/10.1590/2175-3369.009.supl1.ao01.

Givoni, B., Noguchi, M., Saaroni, H., Pochter, O., Yaacov, Y., Feller, N., \& Becker, S. (2003). Outdoor comfort research issues. Energy and Building, 35(1), 77-86. http://dx.doi.org/10.1016/S0378-7788(02)00082-8.

Google Inc. (2018). Google Earth PRO. Versão 7.3.1.4507. Recuperado em 24 de maio de 2017, de https://www.google.com.br/earth/download/gep/agree.html

Herrmann, J., \& Matzarakis, A. (2011). Mean radiant temperature in idealised urban canyons-examples from Freiburg, Germany. International Journal of Biometeorology, 56(1), 199-203.

Höppe, P. (1999). The physiological equivalent temperature - a universal index for the biometeorological assessment of the thermal environment. International Journal of Biometeorology, 43(2), 71-75.

http://dx.doi.org/10.1007/s004840050118. PMid:10552310.

International Organization for Standardization - ISO. (1995). ISO 10551: Ergonomics of the thermal environment: assessment of the influence of the thermal environment using subjective judgment scales. Genève: ISO.

International Organization for Standardization - ISO. (1998). ISO 7726: Ergonomics of the thermal environments Instruments for measuring physical quantities. Genève: ISO. 
International Organization for Standardization - ISO. (2005). ISO 7730: Ergonomics of the thermal environments Analytical determination and interpretation of thermal comfort using calculation of the PMV and PPD indices and local thermal comfort criteria. Genève: ISO.

Jamei, E., Rajagopalan, P., Seyedmahmoudian, M., \& Jamei, Y. (2016). Review on the impact of urban geometry and pedestrian level greening on outdoor thermal comfort. Renewable \& Sustainable Energy Reviews, 54, 1002-1017. http://dx.doi.org/10.1016/j.rser.2015.10.104.

Kruger, E., \& Drach, P. (2017). Quantificação dos impactos da climatização artificial na sensação térmica de transeuntes em termos de alterações no microclima. Urbe. Revista Brasileira de Gestão Urbana, 9(1, suppl 1), 301312. http://dx.doi.org/10.1590/2175-3369.009.supl1.ao04.

Kruger, E., \& Tamura, C. (2015). Urban climate studies in a subtropical location: literature review and current perspectives for Curitiba, Brazil. Energy and Emission Control Technologies, 3, 55-66. http://dx.doi.org/10.2147/EECT.S60290.

Labaki, L. C., Fontes, M. S. G., Bueno-Bartholomei, C. L., \& Dacanal, C. (2012). Conforto térmico em espaços públicos de passagem: estudos em ruas de pedestres no estado de São Paulo. Ambiente Construído, 12(1), 167-183. http://dx.doi.org/10.1590/S1678-86212012000100003.

Lau, K. K.-L., Chung, S. C., \& Ren, C. (2019). Outdoor thermal comfort in different urban settings of sub-tropical highdensity cities: an approach of adopting local climate zone (LCZ) classification. Building and Environment, 154, 227-238. http://dx.doi.org/10.1016/j.buildenv.2019.03.005.

Lee, H., \& Mayer, H. (2018). Thermal comfort of pedestrians in an urban street canyon is affected by increasing albedo of building walls. International Journal of Biometeorology, 62(7), 1199-1209. http://dx.doi.org/10.1007/s00484-018-1523-5. PMid:29532254.

Lindberg, F., Thorsson, S., Rayner, D., \& Lau, K. (2016). The impact of urban planning strategies on heat stress in a climate-change perspective. Sustainable Cities and Society, 25, 1-12. http://dx.doi.org/10.1016/j.scs.2016.04.004.

Masiero, E., \& Souza, L. C. L. (2018). Mapping humidity plume over local climate zones in a high-altitude tropical climate city, Brazil. Ambiente Construído, 18(4), 177-197. http://dx.doi.org/10.1590/s1678-86212018000400300.

Matzarakis, A., Rutz, F., \& Mayer, H. (2000). Estimation and calculation of the mean radiant temperature within urban structures. In: R. J. de Dear, J. D. Kalma, T. R. Oke, \& A. Auliciems (Eds) Biometeorology and Urban Climatology at the Turn of the Millenium : selected papers from the conference ICB-ICUC'99, (pp. 273-278). Sydney: WCASP, WMO/TD.

Middel, A., Häb, K., Brazel, A. J., Martin, C., \& Guhathakurta, S. (2014). Impact of urban form and design on midafternoon microclimate in Phoenix Local Climate Zones. Landscape and Urban Planning, 122, 16-28. http://dx.doi.org/10.1016/j.landurbplan.2013.11.004.

Middel, A., Selover, N., Hagen, B., \& Chhetri, N. (2016). Impact of shade on outdoor thermal comfort: a seasonal field study in Tempe, Arizona. International Journal of Biometeorology, 60(12), 1849-1861.

http://dx.doi.org/10.1007/s00484-016-1172-5. PMid:27192997.

Monteiro, L. M., \& Alucci, M. P. (2007). Conforto Térmico em Espaços Abertos com Diferentes Abrangências Microclimáticas. Parte 2: proposição de calibração de modelos preditivos. In Anais do IX Encontro Nacional e V Encontro Latino-americano sobre Conforto no Ambiente Construído (pp. 1231-1240). Ouro Preto: ANTAC.

Muniz-Gäal, L. P., Pezzuto, C. C., Carvalho, M., \& Mota, L. T. M. (2018). Parâmetros urbanísticos e o conforto térmico de cânions urbanos: o exemplo de Campinas, SP. Ambiente Construído, Porto Alegre, 18(2), 177-196. http://dx.doi.org/10.1590/s1678-86212018000200249.

Ng, E., \& Cheng, V. (2012). Urban human thermal comfort in hot and humid Hong Kong. Energy and Building, 55, 51-65. http://dx.doi.org/10.1016/j.enbuild.2011.09.025.

Nobre, C. A. (Coord.) (2011). Vulnerabilidades das megacidades brasileiras às mudanças climáticas: região metropolitana de São Paulo: relatório final. São José dos Campos, SP: INPE. 
Oke, T. R. (1981). Canyon geometry and the urban heat island. Journal of Climatology, 1(3), 237-254.

http://dx.doi.org/10.1002/joc.3370010304.

Oke, T. R. (1987). Boundary layer climates. (2nd ed.). London: Taylor \& Francis Group.

Perera, N. G. R., \& Emmanuel, R. A. (2016). “Local Climate Zone” based approach to urban planning in Colombo, Sri Lanka. Urban Climate, 23, 188-203. http://dx.doi.org/10.1016/j.uclim.2016.11.006.

Rutz, F., Matzarakis, A., \& Mayer, H. (2000). RAYMAN. Versão 1.2. Germany: Meteorological Institute of the University of Freiburg. Recuperado em 24 de maio de 2017, de https://www.urbanclimate.net/rayman/.

Santamouris, M. (2015). Regulating the damaged thermostat of the cities - Status, impacts and mitigation challenges. Energy and Building, 91, 43-56. http://dx.doi.org/10.1016/j.enbuild.2015.01.027.

São Carlos. Prefeitura Municipal. (2016, 19 de dezembro). Lei 18.053 de 19 de dezembro 2016. Estabelece o Plano Diretor do Município de São Carlos. São Carlos: Diario Oficial. Recuperado em 24 de maio de 2017, de http://www.saocarlosoficial.com.br/diariooficial/001/DO_28122016_HNGB66.pdf

Skarbit, N., Stewart, I. D., Unger, J., \& Gál, T. (2017). Employing an urban meteorological network to monitor air temperature conditions in the 'local climate zones' of Szeged, Hungary. International Journal of Climatology, 37(1), 582-596. http://dx.doi.org/10.1002/joc.5023.

Sobral, H. R. (2005). Heat island in São Paulo, Brazil: effects on health. Critical Public Health, 15(2), 147-156. http://dx.doi.org/10.1080/09581590500151756.

Souza, L. C. L., Tente, C. M., Giunta, M. B., \& Nakata, C. M. (2010). Fator de visão do céu e intensidade de ilhas de calor na escala do pedestre. Ambiente Construído, 10(4), 155-167. http://dx.doi.org/10.1590/S167886212010000400011.

Stewart, I. D., \& Oke, T. R. (2012). Local climate zones for urban temperature studies. Bulletin of the American Meteorological Society, 93(12), 1879-1900. http://dx.doi.org/10.1175/BAMS-D-11-00019.1.

Tsoka, S. (2017). Investigating the Relationship Between Urban Spaces Morphology and Local Microclimate: A Study for Thessaloniki. Procedia Environmental Sciences, 38, 674-681.

http://dx.doi.org/10.1016/j.proenv.2017.03.148.

Unger, J., Skarbit, N., \& Gál, T. (2018). Evaluation of outdoor human thermal sensation of local climate zones based on long-term database. International Journal of Biometeorology, 62(2), 183-193.

http://dx.doi.org/10.1007/s00484-017-1440-z. PMid:28889179.

Wei, R., Song, D., Wong, N. H., \& Martin, M. (2016). Impact of urban morphology parameters on microclimate. Procedia Engineering, 169, 142-149. http://dx.doi.org/10.1016/j.proeng.2016.10.017.

\section{Editor: Harry Bollmann}

Recebido: Set. 03, 2019

Aprovado: Fev. 13, 2020 